\title{
Direct costs of glaucoma: Relationship between cost and severity of the disease
}

Chronic Illness $0(0)$ 1-9

(C) The Author(s) 2018 Article reuse guidelines: sagepub.com/journals-permissions DOI: I0.I I 77//7423953|8803660 journals.sagepub.com/home/chi

(SAGE

\author{
JP Real', MC Lafuente ${ }^{2}$, SD Palma' and \\ LI Tártara'
}

\begin{abstract}
Objectives: To estimate the direct medical costs associated with the management of patients with primary open-angle glaucoma and to compare the costs of patients according to the degree of severity.

Methods: A longitudinal retrospective study was carried out using all patients with primary open-angle glaucoma that recorded follow-up from May 2010 to June 2013 at the Hospital Privado de Córdoba. We estimated the cost of the disease from the perspectives of the institution, with a bottom-up approach.

Results: The three-year follow-up after treatment of 104 patients revealed that the average cost of care for a patient with primary open-angle glaucoma was US\$2746 \pm I 560 . The first year of treatment was significantly more expensive than subsequent ones (US\$1 I00-\$8I0-\$827). Cost was related to the degree of severity of glaucoma; patients in "Stage 0" had significantly lower costs than those in other groups (Kruskal-Wallis test, $p<0.0 \mathrm{I}$ ). This was a consequence of lower costs associated with medication and a lower percentage of patients undergoing surgery. Discussion: The direct medical costs of a patient with primary open-angle glaucoma vary according to the severity of their disease and the year of treatment. We found that costs increased with disease severity, but decreased over time.
\end{abstract}

\section{Keywords}

Glaucoma, cost of illness, health expenditures, severity of illness index, observational studies as topic

Received 22 April 2018; accepted 4 September 2018

\footnotetext{
'UNITEFA-CONICET, Departamento de Farmacia, Facultad de Ciencias Químicas, Universidad Nacional de Córdoba, Córdoba, Argentina

${ }^{2}$ Hospital Privado Universitario Centro Médico de Córdoba, Córdoba, Argentina
}

\section{Corresponding author:}

LI Tártara, Universidad Nacional de Córdoba, Haya de la Torre y Medina Allende, Córdoba, Argentina.

Email: i.tartara@gmail.com 


\section{Introduction}

Glaucoma refers to a group of diseases which have in common an optic neuropathy with degeneration of ganglion cells and loss of visual field (VF). An important risk factor involved in the disease is the increase in intraocular pressure (IOP). ${ }^{1}$

There are more than 60 million people in the world who suffer from primary open angle glaucoma (POAG), and it is estimated that by 2020 there will be 80 million patients with this condition. ${ }^{2}$ It has been shown that patients with glaucoma who do not receive adequate treatment have a chronic and progressive damage at the level of the optic nerve, which leads quickly and inexorably to blindness. ${ }^{3}$ Glaucoma is the second cause of blindness in the world ${ }^{4}$ and the first when it comes to irreversible blindness. ${ }^{5}$

The treatment of glaucoma aims to reduce the elevated IOP and thus prevent or delay the progression of damage to the optic nerve. Currently, there are three types of treatments: pharmacotherapy (topical in most cases), treatments with different types of lasers and surgical procedures. ${ }^{4,6,7}$ Pharmacological treatment includes five types of drugs with various actions: those that act by decreasing the secretion of aqueous humor (beta-blocker-BB and carbonic anhydrase inhibitors-IAC), those that increase the outflow of aqueous humor via uveoscleral or unconventional (Adrenergic agonists-AA and prostaglandin analoguesAPG), and those that increase the output via trabecular or conventional (parasympathomimetic-PSM). ${ }^{8}$ The damage that glaucoma produces is irreversible, and therefore the sooner the diagnosis is made and the appropriate treatment is established, the better the results and the patients' functional vision. ${ }^{\text {? }}$

The treatment of glaucoma as well as its resulting potential blindness implies costs for society and must be individualized to satisfy the educational and socioeconomic aspect of each patient. ${ }^{10}$ The social and economic burden of this disease is higher in developed countries due to longer life expectancy, a higher age profile of the population, and a higher per capita gross domestic product. ${ }^{10}$ As a result of the scarcity of health resources and greater pressure for the efficient use of available resources, studies evaluating the economic impact of diseases and their treatment are of growing interest. There are few studies that investigate the real costs of treatment for the different stages of glaucoma, the impact of the progression of the disease and the economic burden that different patients endure. The objective of this study was to estimate the direct medical costs associated with the management of patients with open-angle glaucoma in an ophthalmology service and to compare the associated costs according to the degree of severity.

\section{Materials and methods}

A retrospective study was performed using all patients with a confirmed diagnosis of POAG who recorded follow-up in the Ophthalmology Service of the Private Hospital of Córdoba (HPC) during the first semester of 2010 and who were attended until June 2013 inclusive.

\section{Inclusion criteria}

Patients older than 18 years with confirmed diagnosis of primary open-angle glaucoma who attended the HPC Ophthalmology Service from May 2010 to June 2013. POAG was defined in patients with increased IOP and progressive decrease in the neuroretinal ring of optic nerve.

\section{Exclusion criteria}

Primary angle-closure glaucoma, congenital glaucoma, and secondary glaucoma. The cost of the disease was estimated using 
the prevalence approach. The average unit cost for each patient was calculated from the third-party payer's perspective. In order to estimate direct health costs, the micro-costing technique (bottom-up approach) was applied. This technique quantifies the resources in a disaggregated manner for each patient based on records review (in this case the internal history of the hospital) for each patient.

We obtained the prices of the different resources used from the following databases:

- The national indicative nomenclature of the Argentine Council of Ophthalmology (CAO, 2015). ${ }^{11}$ This nomenclature was made based on economic and financial calculations with the aim of providing a tool that guarantees the good quality of the ophthalmological medical services.

- The online Pharmaceutical Manual of the Editorial Alfabeta (www.alfabeta. net; 2015).

Costs were expressed in US dollars (U \$A) according to quotes from July, 2015.

We reviewed clinical histories for the following services:

\section{- Ophthalmological consultation: US \$15}

This includes: anamnesis, examination of eyes, eyelids and conjunctiva, medical treatment (example: conjunctivitis, chalazion, pterigion), indicate treatments or diagnostic studies.

\section{- Ophthalmological studies module: US \$28}

This includes: Visual acuity examination (Snellen Optotype), biomicroscopy (TOPCON Slit Lamp), fundus (Slit lamp+ 78 dioptric magnifying glass), tonometry (Perkins Manual Tonometer), gonioscopy, exophthalmometry, indirect binocular ophthalmoscopy, chromatic vision (Ishihara test).

\section{- Associated complementary studies:}

Corneal pachymetry

US $\$ 36$

Standard automated perimetry US $\$ 172$

Confocal laser tomography US $\$ 128$

Daily pressure curve US $\$ 117$

\section{- Associated treatments:}

$\begin{array}{ll}\text { Laser therapy } & \text { US } \$ 306 \\ \text { Surgical } & \text { US } \$ 1120\end{array}$

To determine the cost of pharmacotherapy, it was necessary to obtain the average price of the different commercial presentations (see Table 1) and to determine cost per month according to the use of each patient.

From the clinical point of view, when characterizing the baseline sample (a patient's first visit to the ophthalmologist), the following variables were taken into account: age (in years); sex (expressed as female/male); IOP ( $\mathrm{mmHg})$; the excavation of the optic nerve (expressed in decimal unit); and finally the VF to determine the different stages of the disease, taking into account the classification of Mills et al. ${ }^{12}$ which uses the mean deviation (MD) of the computed VFs (Humphrey):

- Stage 0: $>0.00$

- Early glaucoma: -0.00 to -6

- Moderate glaucoma: -6.00 to -12

- Advanced glaucoma: -12.00 to -20

- Severe glaucoma: -20.00 or worse

In this work, the Advanced and Severe stage (MD $-12 \mathrm{~dB}$ ) were regrouped in the same stage and are collectively referred to as Advanced. 
Table I. Mean price of drugs (used alone and in combinations) according to composition.

\begin{tabular}{llll}
\hline Drug & Price US\$ & Drug & Price US\$ \\
\hline Acetazolamida & $\$ 10.77$ & Brinzolamida+timolol & $\$ 15.12$ \\
Betaxolol & $\$ 5.89$ & Travoprost+timolol & $\$ 16.36$ \\
Betaxolol 0.25\% & $\$ 10.77$ & Timolol, maleato & $\$ 4.64$ \\
Bimatoprost & $\$ 12.85$ & Dorzolamida+timolol & $\$ 13.02$ \\
Bimatoprost+timolol & $\$ 12.78$ & Travoprost & $\$ 9.01$ \\
Brimonidina & $\$ 9.37$ & Latanoprost & $\$ 11.13$ \\
Brimonidina+timolol & $\$ 10.64$ & Latanoprost + timolol & $\$ 10.68$ \\
Brinzolamida & $\$ 11.41$ & Pilocarpina & $\$ 5.73$ \\
\hline
\end{tabular}

The statistical analysis was carried out using the Infostat statistical package (version 2014). For continuous quantitative data, means, standard deviations and standard errors were calculated. In the case of discrete quantitative variables, the quantity in absolute values was measured in addition to the corresponding relative frequency.

For comparisons of quantitative variables, Student's $t$ tests were used for independent and paired samples as well as non-parametric Mann-Whitney and Wilcoxon tests, when the variables did not meet the normality criteria (Shapiro-Wilk test). For the comparison of proportions, the Irwin-Fisher test was used. The Kruskal-Wallis nonparametric analysis of variance was used to compare cost of different stage of glaucoma. A $p$-value of 0.05 or less was considered to be statistically significant. The project was approved by the Institutional Ethics Committee of the Teaching Department of the Private Hospital of Córdoba, which waived the requirement of informed consent because research activities were limited to the confidential review of medical records (Approval number: 2015/02).

\section{Results}

A total of 104 patients were studied, of which $64(62 \%)$ were female. The patients had a mean age of $70.4 \pm 9.45$ years (range: $38-94$ years) with most (73\%) falling within the 60-79 year age group. From the ophthalmological point of view, patients were classified by their eye with the most advanced glaucoma state at baseline. The relevant baseline ophthalmological clinical features are summarized in Table 2.

The analysis of the medication and the treatments (Table 3) showed that $66 \%$ of patients used more than one active ingredient to control their glaucoma, and that Timolol is the most widely used active ingredient.

In general, we observed that the most frequently used pharmacological treatment was the combined use of an APG with a BB and an IAC, with the combination of Latanoprost, Dorzolamide, and Timolol being the treatment of choice in these cases. In the case of patients who used a single active agent for the control of IOP, APGs (especially Latanoprost, used by 22 patients) were the drugs of choice (Table 3 ). Among patients who used more than one active ingredient to control their glaucoma, $48 \%$ used a single eye drop with a fixed combination of drugs.

Regarding the direct health costs involved in the treatment of selected patients, our analysis showed that the total cost of care for the 104 patients studied over those three years was US \$284,971. The mean cost $( \pm \mathrm{SD})$ of three years of treatment for a patient with POAG in the service was US $\$ 2746 \pm 1560$ (range: US 
Table 2. Clinical characteristics of the worst eye in the baseline follow-up (2010).

\begin{tabular}{lccccc}
\hline Patient group & $\mathrm{N}$ & $\begin{array}{l}\text { Excavation } \\
(\operatorname{mean} \pm S D)\end{array}$ & $\begin{array}{l}\text { IOP } \\
(\text { mean } \pm S D)\end{array}$ & $\begin{array}{l}\text { MD } \\
(\text { mean } \pm S D)\end{array}$ & $\begin{array}{l}\text { LV } \\
(\text { mean } \pm S D)\end{array}$ \\
\hline Stage 0 & 21 & $0.33 \pm 0.13$ & $14.86 \pm 2.69$ & $+1.16 \pm 0.71$ & $2.96 \pm 2.08$ \\
Early glaucoma & 41 & $0.49 \pm 0.21$ & $14.01 \pm 1.88$ & $-2.49 \pm 1.82$ & $15.18 \pm 16.7$ \\
Moderate glaucoma & 21 & $0.51 \pm 0.23$ & $14.84 \pm 1.95$ & $-8.27 \pm 1.39$ & $39.82 \pm 18.52$ \\
Advanced glaucoma & 21 & $0.66 \pm 0.24$ & $14.45 \pm 3.67$ & $-18.9 \pm 4.11$ & $44.12 \pm 27.81$ \\
Total average & 104 & $0.5 \pm 0.23$ & $14.44 \pm 2.48$ & $-5.91 \pm 7.38$ & $22.67 \pm 23.83$ \\
\hline
\end{tabular}

Note: Patients grouped by severity of glaucoma according to Mills et al. ${ }^{12}$ classification.

IOP: intraocular pressure; MD: mean deviation; LV: lost variance; SD: Standard deviation.

Table 3. Medication and treatments used by patients.

\begin{tabular}{|c|c|c|c|c|}
\hline \multicolumn{3}{|c|}{$\begin{array}{l}\text { Pharmaceutically active ingredient (PAI) } \\
\text { used according to frequency of use }\end{array}$} & \multicolumn{2}{|c|}{$\begin{array}{l}\text { Distribution of patients according } \\
\text { to the treatment used }\end{array}$} \\
\hline PAI & Therapeutic Group & Percentage (\%) & Treatment & $N$ \\
\hline Timolol & BB & 73 & $A P G^{a}$ & 23 \\
\hline Latanoprost & APG & 62 & $\mathrm{BB}^{\mathrm{a}}$ & 10 \\
\hline Dorzolamida & IAC & 33 & $I A C^{a}$ & 2 \\
\hline Travoprost & APG & 20 & $\mathrm{AA}^{\mathrm{a}}$ & 1 \\
\hline Brinzolamida & IAC & 13 & $A P G+I A C+B B$ & 34 \\
\hline Brimonidina & $\mathrm{AA}$ & & $\mathrm{APG}+\mathrm{BB}$ & 19 \\
\hline Betaxolol & BB & 3 & $\mathrm{IAC}+\mathrm{BB}$ & 6 \\
\hline Carteolol & BB & 3 & $\mathrm{APG}+\mathrm{AA}$ & 4 \\
\hline Bimatoprost & APG & 2 & $A A+I A C+B B$ & 2 \\
\hline Acetazolamida & IAC & 2 & $\mathrm{APG}+\mathrm{AA}+\mathrm{BB}$ & I \\
\hline Levobunolol & BB & I & $\mathrm{APG}+\mathrm{SPM}+\mathrm{BB}$ & I \\
\hline Pilocarpina & PSM & I & $A P G+I A C+A A$ & I \\
\hline
\end{tabular}

a Monotherapy.

BB: beta-blocker; IAC: carbonic anhydrase inhibitor; APG: prostaglandin analogue; PSM: parasympathomimetics; AA: adrenergic agonists.

$\$ 424$ to $\$ 11,445)$. The average cost of the first year of treatment was significantly higher than the subsequent years (Student's $t$-test for paired samples, $p<0.01$, see Figure 1).

This decrease in costs is explained by a significant reduction in the number of consultations (see Table 4), complementary studies and surgeries (see Figure 2).

Contrary to the other costs evaluated, the costs associated with medication, which represent $46 \%$ of the total costs, increased significantly each year with a mean $( \pm$ SD) cost of US $\$ 506 \pm 261, \$ 546$ \pm 267 and $\$ 552 \pm 299$ for the first, second and third year, respectively (Figure 1).

When patients were grouped by glaucoma stage, differences were observed between the costs amassed by the different groups (see Figure 3); costs associated with "Stage 0" were significantly lower than the rest (Kruskal-Wallis test, $p<0.01$ ).

The greatest differences were in the pharmacological treatment and the percentage 


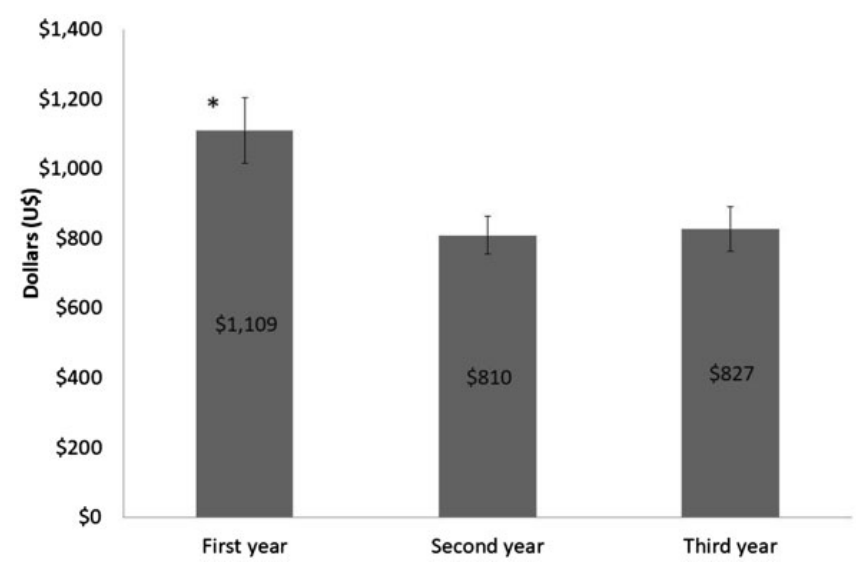

Figure I. Average direct health costs per year of a patient with primary open-angle glaucoma (POAG). *Statistically superior to subsequent years. Statistic: T test (paired samples) Dollar-Peso Price July 2015.

Table 4. Mean consultations per year of patients with primary open-angle glaucoma.

\begin{tabular}{lllll}
\hline Consultations & AVG & SD & LL (95) & UL (95) \\
\hline Year I & 3.69 & 2.9 & 3.13 & 4.26 \\
Year 2 & 1.85 & 1.74 & 1.51 & 2.19 \\
Year 3 & 1.78 & 1.67 & 1.45 & 2.1 \\
\hline
\end{tabular}

AVG: average; SD: standard deviation; LL and UL: lower and upper limit of $95 \%$ confidence intervals.

of patients who undergo surgery for glaucoma (see Figure 4).

\section{Discussion}

Knowing the benefits and costs associated with the treatment of the visual diseases has health and economic impacts for society as a whole, since treatment costs constitute the basis for the formulation of health policies that are used to efficiently allocate means. In the treatment of chronic and progressive diseases such as glaucoma, it could be postulated that as the severity of the disease worsens, the consumption of resources should increase in order to slow down the disease's progression. In particular, glaucoma is usually asymptomatic in the early stages, resulting in late diagnosis and relatively intensive medical attention during the later stages of the disease. The results of this study support the hypothesis that in patients with glaucoma, the use of resources and treatment costs increases as the disease worsens. The total cost of institutional care for the 104 patients with POAG included in our study was US $\$ 284,971$ with the annual cost ranging from US $\$ 688$ for each patient with stage 0 glaucoma and US $\$ 1079$ for patients with advanced glaucoma. The study conducted by Lee et al. ${ }^{13}$ shows that in the United States, the average annual direct cost for an early stage patient is similar to that of our data (US \$623); however, the cost calculated for the advanced stages is significantly higher than those recorded in our present study (US \$2464). The differences observed between the two studies might be explained by greater access to complementary studies and surgeries in the US compared to Argentina. It should be noted that the costs associated with visual rehabilitation and either formal or informal care were not considered. It is expected that the more advanced the disease is, the higher the costs will be. To further support the findings of this study, new 


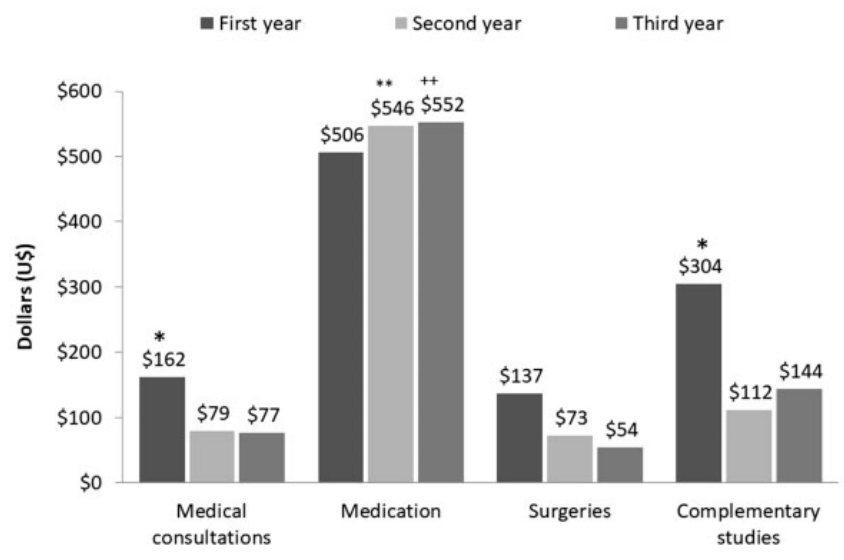

Figure 2. Average direct health costs per item per year of a patient with primary open-angle glaucoma. *Statistically superior to subsequent years. **Statistically superior to first year. ++ Statistically superior to first and second year Statistic: T test (paired samples) Dollar-Peso Quote July 2015.

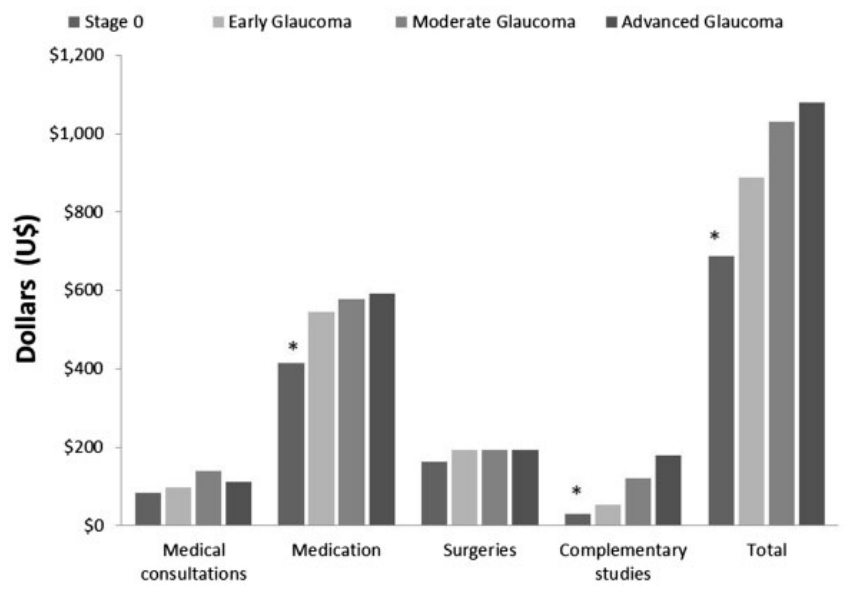

Figure 3. Average direct health costs per year by sector according to glaucoma severity status according to the classification of Mills et al. ${ }^{12}$ *Statistically lower than the average cost of the other states. Statistics: Kruskal-Wallis test. Dollar-Peso Price July 2015.

studies from a social perspective should be considered. Examining costs from a social perspective, as opposed to the payer's perspective, can have a significant impact on the treatment costs, especially for patients with advanced disease in which the nonreimbursable costs of low vision rehabilitation are higher.

In a study by Koleva et al., ${ }^{14}$ on institutional costs of glaucoma and ocular hypertension, the average number of consultations per year for patients with glaucoma was 3.68. We obtained similar data regarding the number of consultations: 3.7 consultations in the first year, decreasing to 1.8 consultations in the following years. At the beginning of treatment, it is likely that both physicians and patients comply with the recommendations of frequency of medical consultation until an exhaustive 


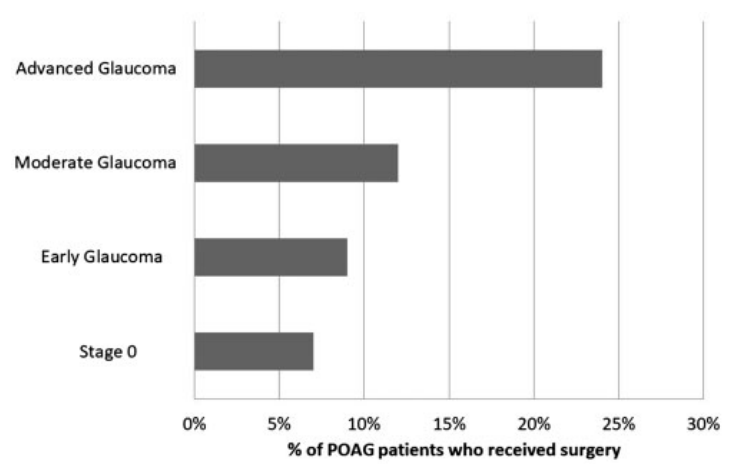

Figure 4. Proportion of patients from the analyzed cohort who underwent surgery in the evaluated period of time grouped by severity of primary open-angle glaucoma. There were no significant differences between the Groups. Statistic: Test Irwin-Fisher.

examination of the patient is carried out. It is likely that this consistency or adhesion will decrease over time, which would lead to a significant reduction in annual consultations.

In this work, we found that medications are the main factor in the total direct cost of glaucoma; they represent between 55 and $61 \%$ of the total direct cost across all levels of severity. Incomplete coverage of these medications by health insurers implies that patients must assume 25 to $30 \%$ of the total treatment costs. These costs are added to the costs associated with the transportation to visits and the costs of coinsurance needed to receive certain benefits. Studies of the cost of illness in other countries have shown that the patient's out-of-pocket payment can impact therapy adherence and persistence. ${ }^{6}$ Adherence and persistence are very important factors to ensure the effectiveness of the treatment and therefore visual morbidity. ${ }^{3}$ In our study, there was a decrease in medical consultations over time associated with increases in medication and worsening of the pathology. Some authors warn that low therapy adherence is associated with worse disease control, which triggers incremental pharmacotherapy with increasing doses of medication, ultimately increasing costs and the risk of adverse effects. ${ }^{15}$ In other words, the phenomena observed could correspond to a decrease in the therapeutic adherence of the patient.

In conclusion, the direct medical costs of a patient with open-angle glaucoma vary according to the degree of severity of their pathology and the year of treatment. According to the present results, costs increase with disease severity worsening, perhaps due to disease progression among nonadherent patients.

\section{Acknowledgements}

We appreciate the collaboration provided by the Ophthalmology Service of the Hospital Privado of Cordoba. This paper has been professionally proofread by Proof-Reading-Service.com.

\section{Declaration of conflicting interests}

The author(s) declared no potential conflicts of interest with respect to the research, authorship, and/or publication of this article.

\section{Ethical approval}

This study complied with the Helsinki declaration and the law 25326/2000 "Protection of Personal Data" that ensures the confidentiality of information obtained and the identity of patients involved. 


\section{Ethical considerations}

The project was approved by the Institutional Ethics Committee of the Teaching Department of the Private Hospital of Cordoba, which waived the requirement of informed consent because the research activities were limited to the confidential review of medical records (Approval number: 2015/02).

\section{Funding}

The author(s) received no financial support for the research, authorship, and/or publication of this article.

\section{Informed consent}

The Ethical Board at the Institutional Ethics Committee of the Teaching Department of the Private Hospital of Córdoba determined that informed consent was not required.

\section{References}

1. García Alcolea E and Ortiz González E. Blindness prevention in patients with primary open angle glaucoma. Rev haban cienc méd 8(3), http://scielo.sld.cu/scielo.php? script = sci_arttext\&p id = S1729519X2009000300004\&lng $=$ es\&nrm $=$ iso (2009, accessed 18 May 2018).

2. Quigley $\mathrm{H}$ and Broman A. The number of people with glaucoma worldwide in 2010 and 2020. $\mathrm{Br} \quad J$ Ophthalmol 2006; 90: 262-267.

3. Chae B, Cakiner-Egilmez T and Desai M. Glaucoma medications. Insight 2013; 38: 5-9.

4. Kumbar S, Mirje M, Moharir G, et al. Cost analysis of commonly used combination of drugs in primary open angle glaucoma. J Clin Diagn Res 2015; 9: 5-8.

5. Varma R, Ying-Lai M, Francis B, et al. Prevalence of open-angle glaucoma and ocular hypertension in Latinos: the Los
Angeles Latino eye study. Ophthalmology 2004; 111: 1439-1448.

6. Tártara IJ, Kairuz A, Allemandi DA, et al. Uso racional de tecnologías sanitarias: tratamiento del glaucoma. Latin Am J Pharm 2008; 27: 297-302.

7. Stein JD, Kim DD, Peck WW, et al. Costeffectiveness of medications compared with laser trabeculoplasty in patients with newly diagnosed open-angle glaucoma. Arch Ophthalmol 2012; 130: 497-505.

8. $\mathrm{Li} \mathrm{T}$, Lindsley $\mathrm{K}$, Rouse $\mathrm{B}$, et al. Comparative effectiveness of first-line medications for primary open-angle glaucoma: a systematic review and network meta-analysis. JAMA Ophthalmol 2016; 123: 129.

9. Nouri-Mahdavi $\mathrm{K}$ and Caprioli J. Measuring rates of structural and functional change in glaucoma. $\mathrm{Br} J$ Ophthalmol 2015; 99: 893-898.

10. Nayak B, Gupta S, Kumar G, et al. Indian socioeconomics of long-term glaucoma therapy in India. Ind $J$ Ophthalmol 2015; 63: 20-24.

11. Consejo Argentino de Oftalmología [CAO]. Nomenclador Nacional Indicativo, http:// oftalmologos.org.ar/servicios/nomenclador (accessed 15 June 2015).

12. Mills R, Budenz D, Lee $P$, et al. Categorizing the stage of glaucoma from pre-diagnosis to end-stage disease. $\mathrm{Am} J$ Ophthalmol 2006; 141: 24-30.

13. Lee PP, Walt JG, Doyle JJ, et al. A multicenter, retrospective pilot study of resource use and costs associated with severity of disease in glaucoma. Arch Ophthalmol 2006; 124: $12-19$.

14. Koleva D, Motterlini I, Schiavone M, et al. Medical costs of glaucoma and ocular hypertension in Italian referral centres: a prospective study. Ophthalmologica 2007; 221: 340-347.

15. Bosworth HB, et al. Medication adherence: a call for action. Am Heart $J$ 2011; 162: 412-424. 